Research Article

\title{
Functional Evaluation of the Visual Pathway in Patients with Multiple Sclerosis Using a Multifunction Stimulator Monitor
}

\author{
M. Satue $\mathbb{D}^{1,},{ }^{1,2}$ L. Jarauta, ${ }^{3}$ J. Obis $\mathbb{D}^{1,2}$ M. Cipres, ${ }^{1,2}$ M. J. Rodrigo ${ }^{1},{ }^{1,2}$ C. Almarcegui, ${ }^{2,4}$ \\ I. Dolz, ${ }^{2,4}$ J. R. Ara, ${ }^{2,3}$ J. Martin, ${ }^{2,3}$ L. E. Pablo, ${ }^{1,2}$ and E. Garcia-Martin $\mathbb{D}^{1,2}$ \\ ${ }^{1}$ Ophthalmology Department, Miguel Servet University Hospital, Zaragoza, Spain \\ ${ }^{2}$ IIS-Aragon, Aragon Health Research Institute, Zaragoza, Spain \\ ${ }^{3}$ Neurology Department, Miguel Servet University Hospital, Zaragoza, Spain \\ ${ }^{4}$ Neurophysiology Department, Miguel Servet University Hospital, Zaragoza, Spain
}

Correspondence should be addressed to M. Satue; mariasatue@gmail.com

Received 24 February 2019; Accepted 30 July 2019; Published 18 September 2019

Academic Editor: Inés Contreras

Copyright $\odot 2019$ M. Satue et al. This is an open access article distributed under the Creative Commons Attribution License, which permits unrestricted use, distribution, and reproduction in any medium, provided the original work is properly cited.

\begin{abstract}
Objectives. To assess the capability of the vision monitor unit Monpack One of detecting visual function alterations in patients with multiple sclerosis (MS) and to evaluate the correlation between structural retinal parameters and functional measurements obtained with this device. Methods. Forty-eight patients with MS and 46 healthy controls were included in a cross-sectional study. All participants underwent a complete functional evaluation of the visual pathway, which included low-contrast visual acuity (LCVA), contrast sensitivity vision (CSV), automated perimetry, multifocal visual evoked potentials (mfVEPs), and pattern electroretinogram (ERG). All tests were performed using the vision monitor unit Monpack One (Metrovision, France), a multifunction stimulator device. Retinal structural measurements were obtained in all subjects using Triton swept source optical coherence tomography (Topcon, Japan). Results. Patients with MS presented reduced low-contrast VA $(p<0.001)$ and reduced CSV at medium $(p=0.001, p=0.013)$ and low $(p=0.001, p=0.002)$ spatial frequencies. All visual field parameters were found to be altered in MS patients compared with controls $(\leq 0.001)$. Patients with MS presented lower amplitude of the P100 waveform of the mfVEP in areas corresponding to central $(p<0.001)$, inferonasal $(p=0.001)$, and inferotemporal $(p=0.003)$ retina. The pattern ERG did not show significant differences. Significant correlations were observed between structural retinal measurements and functional parameters, especially between the inner macular areas and measurements corresponding to contrast sensitivity and perimetry indexes. Conclusions. Patients with MS present visual dysfunction detectable with the vision monitor unit Monpack One. This device may be a fast and useful tool to provide a full evaluation of axonal damage in patients with multiple sclerosis.
\end{abstract}

\section{Introduction}

Multiple sclerosis (MS) is a multifocal central nervous system disorder characterized by inflammatory demyelinating lesions affecting white and gray matter. Even without a history of optic neuritis (ON), optic nerve atrophy and thinning of the peripapillary retinal nerve fiber layer are two typical findings of patients suffering from MS [1].

Axonal loss is considered to be the main cause of progressive disability in MS [1-3], and neuronal loss is increasingly recognized as a biomarker that correlates with disability in these patients [4-7]. MS is often associated with involvement of the visual pathway that can lead to clinically evident manifestations (such as ON and diplopia) and more frequently, to subclinical alterations. Decreased contrast sensitivity and color vision deficiencies have been widely observed in MS [8-10] and have been correlated with poorer performances in everyday tasks, such as driving and reading.

Previous research on functional evaluation of the visual pathway demonstrated altered responses in MS. Visual evoked potentials (VEPs) and pattern electroretinogram (ERG) show frequent abnormalities in these patients [1113]. Asymptomatic visual field disturbances seem to be present also in MS patients without a previous episode of 
optic neuritis [14]. Recent studies have correlated alterations in functional responses with structural changes in the retina of MS patients [10,11].

In this study, we evaluated the visual pathway of MS patients using the vision monitor unit Monpack One, a multifunction stimulator device which integrates different functional, electrophysiological, and psychophysical tests for the complete evaluation of visual function parameters. The main objective of this study was to assess the capability of this device of detecting visual function abnormalities through different tests (visual acuity, contrast sensitivity vision, perimetry, visual evoked potentials, and pattern electroretinogram) in patients with MS and to analyze the correlation between the functional measurements obtained and retinal structural parameters measured with swept source optical coherence tomography (SS-OCT).

\section{Methods}

Patients with definite relapsing-remitting (RR) MS were included in this observational cross-sectional study. A total of 48 eyes of 48 patients and 46 eyes of 46 healthy individuals were evaluated. All procedures adhered to the tenets of the Declaration of Helsinki, and all participants provided written informed consent to participate in the study.

The diagnosis of MS was based on the McDonald criteria and confirmed by a neurologist [15]. Related medical records were carefully evaluated, and information about Expanded Disability Status Scale (EDSS) scores, disease duration and subtype, and modifying disease treatments and prior episodes of ON was recorded. Only patients with RR MS were included in our study. Patients with a visual acuity $<0.1$ (using Snellen scale), intraocular pressure $>20 \mathrm{mmHg}$, refractive errors $>5$ diopters (D) of equivalent spherical or $3 \mathrm{D}$ of astigmatism, active MS outbreaks (of any neurologic deficit), and/or history of a previous episode of ON were excluded from our study. The reason to exclude patients with a prior $\mathrm{ON}$ episode was that visual function loss secondary to $\mathrm{ON}$ is widely demonstrated, but the main purpose of this study is to check if neuronal damage secondary to MS itself (i.e., chronic neurodegeneration) causes subclinical visual affectation. The diagnosis of $\mathrm{ON}$ was based on reports from the patient and the treating neurologist and clinical findings such as decrease in visual acuity, visual defects in the perimetry, color vision loss, relative afferent pupillary defect, and papillary pallor observed in the funduscopy.

All eyes underwent a complete neuro-ophthalmic evaluation that included pupillary, anterior segment, and funduscopic examination to detect any ocular pathology that might affect visual function tests.

Visual function was evaluated with the vision monitor unit Monpack One (Metrovision, France), a multifunction stimulator device which integrates different functional, electrophysiological, phychophysical, and oculomotor tests for a complete evaluation of visual function parameters. The vision monitor comprises a central liquid crystal display panel with LED backlight and four peripheral panels illuminated with LEDs. A light sensor placed anteriorly provides feedback to the entire system allowing a constant luminance during the test performance. In our study, visual acuity (VA), contrast sensitivity vision (CSV), visual field, multifocal PEV, and pattern ERG were registered and analyzed in all eyes.

Low-contrast VA was evaluated at a contrast level of $10 \%$. The percentage indicates the level of contrast; that is, $100 \%$ would represent black letters over white background, and $10 \%$ represents medium gray letters over white background. Two different ETDRS charts were selected (one chart for each eye) to avoid learning and memory bias. Measurements were obtained under scotopic light conditions, at a distance of 4 meters in monocular vision using best correction. Visual results were recorded and registered as a VA result (expressed in logMar) as well as the number of total read letters.

Static CSV was evaluated using a sinusoidal pattern (grid) at different spatial frequencies: 0.5, 1, 2, 5, 10, and 20 cycles per degree. Threshold CSV was analyzed by progressive increase of $0.25 \mathrm{~dB}$ of contrast before the test performance. For contrast sensitivity evaluation, the patient/ control pressed a push-button when the contrast grid was first visible in each spatial frequency. Measurements were obtained at 2 meters of distance under scotopic light conditions and monocular vision. CSV results were registered as a sensitive curve in $\mathrm{dB}$ (0 representing no contrast sensitivity).

Visual field was assessed using the vision monitor Fast Perimetry 30 protocol (Fast-30), which analyzes 94 points over the central visual field. The test was performed in monocular vision using near distance correction. Fixation was controlled through a central video camera. Quality parameters (i.e., fixation and attention) were analyzed, and tests with poor performances ( $>5 / 15$ fixation losses and $>5$ / 15 attention losses) were automatically rejected by the program. Results were presented as a $2 \mathrm{D}$ and $3 \mathrm{D}$ sensitivity map (in $\mathrm{dB}$ ), a color probability score map, and a sensitivity curve. The average deficit (AD), corrected average deficit (CAD), variance of deficits (VD), and temporal fluctuation (TF) were recorded.

Electrophysiological evaluation included multifocal VEPs and pattern ERG. Multifocal VEPs were assessed using alternate checkboards with a central fixation point. Electrical responses were recorded using four electrodes attached to the subject scalp by subcutaneous needles (inion: $4 \mathrm{~cm}$ above the inion, $1 \mathrm{~cm}$ above the inion, and $4 \mathrm{~cm}$ right/left of the inion). The ground electrode was placed in the subject's vertex. VEPs were evaluated with the MVEP35 procedure, which analyzes 35 zones for each eye. The test was performed at a $33 \mathrm{~cm}$ distance, under scotopic conditions after 2 minutes light adaptation, using near distance correction in monocular vision. Fixation was controlled through a central video camera. Results were presented as analytic data, amplitude histogram, color map, and $3 \mathrm{D}$ representation for 5 different retinal areas: central, superonasal, superotemporal, inferonasal, and inferotemporal. Amplitude of P100 wave for each retinal area was recorded and expressed in $\mathrm{nV} / \mathrm{deg}^{2}$.

The pattern ERG was evaluated using alternate checkboards with a central fixation point. Electrical responses were recorded using a total of 5 DTL (Dawson-Trick-Litzkow) 
electrodes: 2 electrodes (one for the right eye and one for the left eye) draped horizontally across the cornea at the level of the lower lid (prior topical anesthesia) and 2 reference electrodes positioned at the outer canthus (right and left eye). The ground electrode was placed at Fpz. The pattern ERG uses a reversal stimulation pattern covering a field of 60 degrees with large patterns for 50 minutes. The test was performed at a distance of $30 \mathrm{~cm}$, under scotopic conditions after 2 minutes of light adaptation, using near distance correction in binocular vision. Results are expressed as a graphic of the electrical response registered and as the analytic data of the different spikes selected. Amplitude and latency of the N35, P50, and N95 waves (spikes) were recorded.

Structural measurements of the retina were obtained using the DRI Triton SS-OCT device (Topcon, Tokyo, Japan) in all eyes. The 3D wide protocol was used, which includes a wide scanning range that focuses on both the macular (ETDRS: Early Treatment Diabetic Retinopathy Study) and the peripapillary (TSNIT: temporal-superior-nasal-inferiortemporal) area. With the ETDRS scan, full retinal thickness in nine macular areas (which include a central $1 \mathrm{~mm}$ circle representing the fovea, and inner and outer rings measuring $3 \mathrm{~mm}$ and $6 \mathrm{~mm}$ in diameter, respectively) was analyzed; the TSNIT scan provides automated separate measurements of different retinal layers: retinal nerve fiber layer (RNFL), ganglion cell layer (GCL), retinal thickness, and the choroidal plexus. The TSNIT provides measurements of the 4 peripapillary quadrants (superior, nasal, inferior, and temporal), 6 sectors (superonasal, superotemporal, nasal, temporal, inferonasal, and inferotemporal), and 12 clock sectors. Only measurements of the GCL in the 6 peripapillary sectors were evaluated in this study. All scans were obtained by the same experienced operator and were checked by an experienced rater for quality of the segmentation immediately after acquisition. The DRI Triton SS-OCT provides a quality scale in the image to indicate the signal strength. The quality score ranges from 0 (poor quality) to 100 (excellent quality). Only images with a score $>55$ were analyzed in our study; poor quality images were rejected and recaptured prior to data analysis.

All data analyses were performed using SPSS software version 20.0 (SPSS Inc., Chicago, IL). The KolmogorovSmirnov test was used to assess sample distribution. Differences between evaluations of MS patients and healthy subjects were compared using the Mann-Whitney $U$ test, as the sample did not correspond to a normal distribution (Kolmogorov test: $p<0.05$ ). Correlation between functional and structural data of all subjects (patients and controls) was assessed using Spearman's rho test. Correlation between the EDSS score and visual function parameters was also analyzed. Each eye was considered separately, and only one eye from each patient was randomly selected for analysis.

\section{Results}

Forty-eight patients with MS and 46 healthy controls were included in the study. The mean age of the patients with MS was $49.25(\mathrm{SD}=12.98)$, and the mean age of the healthy controls was $45.74(\mathrm{SD}=10.52)$. The female/male ratio was
4/1 in both groups. Age, sex, and intraocular pressure did not differ significantly between healthy controls and patients with MS ( $p=0.154,0.145$, and 0.770 , respectively).

3.1. Visual Function Analysis. All patients had been diagnosed with MS relapse-remitting subtype and were under treatment with interferon (50\%), glatiramer acetate (12.5\%), or fingolimod (8.3\%). Only $29.2 \%$ of the patients were not under any current treatment. The mean EDSS score was 2.03 $(\mathrm{SD}=0.54)$.

Patients with MS presented reduced low-contrast VA $(0.43 \pm 0.50$ in patients vs. $0.08 \pm 0.27$ in controls, $p<0.001)$ and reduced number of read letters $(31.90 \pm 8.20$ letters vs. $39.70 \pm 5.58$ letters, $p<0.001)$ compared with healthy controls (see Table 1).

CSV was found to be reduced in MS patients in all spatial frequencies. CSV at medium frequencies ( $2 \mathrm{cpd}$ : $21.29 \pm 2.95$ in patients vs. $22.06 \pm 2.12$ in controls, $p=0.001 ; 5 \mathrm{cpd}$ : $20.34 \pm 1.68$ vs. $21.91 \pm 1.94, p=0.013)$ and low frequencies $(1 \mathrm{cpd}: 19.09 \pm 1.55$ vs. $20.50 \pm 2.02, p=0.001 ; 0.5 \mathrm{cpd}$ : $15.98 \pm 1.69$ vs. $17.04 \pm 2.03, p=0.002$ ) was significantly reduced in patients compared with controls (see Table 1).

All visual field parameters were found to be altered in MS patients compared with controls. The $\mathrm{AD}(p<0.001)$, the CAD $(p<0.001)$, the VD $(p<0.001)$, and the TF $(p=0.001)$ were significantly reduced in patients compared with healthy subjects (see Table 1).

Patients with MS presented lower amplitude of the P100 waveform in the multifocal VEP. Significant differences between patients and controls were observed in the areas corresponding to central $\left(798.80 \pm 585.58 \mathrm{nV} / \mathrm{deg}^{2}\right.$ in patients vs. $1556.81 \pm 1120.97 \mathrm{nV} / \mathrm{deg}^{2}$ in controls, $p<0.001$ ), inferonasal $\left(523.90 \pm 262.71 \mathrm{nV} / \mathrm{deg}^{2}\right.$ vs. $798.50 \pm 390.14 \mathrm{nV} / \mathrm{deg}^{2}$, $p=0.001)$, and inferotemporal $\left(677.55 \pm 730.19 \mathrm{nV} / \mathrm{deg}^{2} \mathrm{vs}\right.$. $830.40 \pm 380.09 \mathrm{nV} / \mathrm{deg}^{2}, \quad p=0.003$ ) retina (see Table 2). Patients with MS also presented delayed latency in the N35, P50, and N95 waveforms of the pattern ERG. However, significant differences between patients and controls were not found in any of the pattern ERG responses (see Table 2).

Reports from CSV and mfVEPs as provided by Monpack One are seen in Figure 1.

3.2. Structural Evaluation. Patients presented significantly reduced macular retinal thickness $(p<0.001)$ in all evaluated ETDRS areas and reduced peripapillary GCL thickness $(p<0.001)$ in all 6 sectors analyzed, compared to healthy controls (see Supplementary Table available here).

3.3. Correlation Analysis. A significant correlation was observed between macular parameters and functional measurements obtained with the Monpack One device. The inner superior and inner inferior areas correlated most strongly with contrast sensitivity parameters (the strongest correlation was observed between inner superior and $5 \mathrm{cpd}$, $r=0.36, p<0.001)$. All inner sectors of the macular ETDRS ring presented a positive correlation with the number of read letters (superior: $r=0.37, p<0.001$; nasal: $r=0.21, p=0.042$; 
TABLE 1: Visual acuity, contrast sensitivity vision, and visual field parameters (standard deviation) in patients with multiple sclerosis and healthy controls, as measured with the Monpack One device.

\begin{tabular}{|c|c|c|c|}
\hline Functional parameters & MS patients & Controls & $p$ \\
\hline \multicolumn{4}{|l|}{ Contrast vision } \\
\hline VA ETDRS $10 \%$ & $0.43(0.50)$ & $0.08(0.27)$ & $<0.001$ \\
\hline Read letters & $31.90(8.20)$ & $39.70(5.58)$ & $<0.001$ \\
\hline CSV $0.5 \mathrm{cpd}$ & $15.98(1.69)$ & $17.04(2.03)$ & 0.002 \\
\hline CSV 1 cpd & $19.09(1.55)$ & $20.50(2.02)$ & 0.001 \\
\hline CSV 2 cpd & $21.29(2.95)$ & $22.06(2.12)$ & 0.001 \\
\hline CSV 5 cpd & $20.34(1.68)$ & $21.91(1.94)$ & 0.013 \\
\hline CSV $10 \mathrm{cpd}$ & $19.35(2.60)$ & $21.83(2.84)$ & 0.088 \\
\hline CSV $20 \mathrm{cpd}$ & $11.73(4.31)$ & $15.17(3.21)$ & 0.101 \\
\hline \multicolumn{4}{|l|}{ Visual field parameters } \\
\hline Avg. deficit & $0.14(0.35)$ & $1.64(2.87)$ & $<0.001$ \\
\hline Corrected avg. deficit & $0.20(0.46)$ & $1.57(2.49)$ & $<0.001$ \\
\hline Variance of deficits & $4.05(6.03)$ & $14.65(16.52)$ & $<0.001$ \\
\hline Temporal fluctuation & $0.98(0.45)$ & $1.73(1.34)$ & $<0.001$ \\
\hline
\end{tabular}

Bold letters indicate statistical significance $(p<0.05)$. Abbreviations: MS, multiple sclerosis; VA, visual acuity; CSV, contrast sensitivity vision; cpd, cycles per degree; avg., average.

TABLE 2: Electrophysiological parameters (standard deviation) in patients with multiple sclerosis and healthy controls, as measured with the Monpack One device.

\begin{tabular}{|c|c|c|c|}
\hline Electrophysiological parameters & MS patients & Controls & $p$ \\
\hline \multicolumn{4}{|l|}{$m f V E P s\left(n V / d_{e g}^{2}\right)$} \\
\hline Central & $798.80(585.58)$ & $1556.81(1120.9)$ & $<0.001$ \\
\hline Superonasal & $504.00(278.84)$ & $637.95(675.843)$ & 0.455 \\
\hline Superotemporal & $533.31(292.29)$ & $579.58(341.27)$ & 0.610 \\
\hline Inferonasal & $523.90(262.71)$ & $798.50(390.14)$ & 0.001 \\
\hline Inferotemporal & $677.55(730.19)$ & $830.40(380.09)$ & 0.003 \\
\hline \multicolumn{4}{|l|}{ Pattern ERG } \\
\hline N35 latency (msec) & $27.18(29.31)$ & $23.82(5.34)$ & 0.515 \\
\hline N35 amplitude (mV) & $-0.70(1.34)$ & $-0.62(2.45)$ & 0.534 \\
\hline P50 latency (msec) & $46.59(10.05)$ & $43.34(15.17)$ & 0.471 \\
\hline P50 amplitude (mV) & $4.43(4.79)$ & $7.21(11.32)$ & 0.097 \\
\hline N95 latency (msec) & $83.09(21.06)$ & $71.07(25.97)$ & 0.070 \\
\hline N95 amplitude (mV) & $-3.27(5.38)$ & $-4.79(6.30)$ & 0.184 \\
\hline
\end{tabular}

Bold letters indicate statistical significance $(p<0.05)$. Abbreviations: MS, multiple sclerosis; mfVEPs, multifocal visual evoked potentials; ERG, electroretinogram.

inferior: $r=0.27, p=0.009$; and temporal: $r=0.26$, $p=0.011$ ) and an inverse association with all visual field parameters (the strongest correlation was observed between inner superior and VD, $r=0.35, p=0.001)$. Central thickness correlated with the P100 amplitude of central retina measured with the $\operatorname{mfVEP}(r=0.31, p=0.002)$. No correlations were found between functional parameters and the outer macular areas. Results are shown in Tables 3 and 4.

Peripapillary GCL thickness (total and the temporal quadrant) correlated significantly with most functional parameters analyzed with the Monpack One device. A positive correlation was observed between total GCL thickness and CSV at $1,2,5$, and $10 \mathrm{cpd}$ (the strongest correlation at $10 \mathrm{cpd}, r=0.28, p=0.007)$, and a negative association was observed between total GCL thickness and visual field parameters (the strongest correlation with CAD, $r=-0.32, p=0.002)$. A positive correlation between total GCL and the VEP in central retina $(r=0.26, p=0.012)$ was observed. Temporal GCL thickness correlated with CSV at 2,
5,10 , and $20 \mathrm{cpd}$ (the strongest at $10 \mathrm{cpd}, r=0.40, p<0.001$ ) and all visual field parameters (the strongest association with CAD, $r=-0.36, p>0.001)$. Additionally, a significant relationship was observed between GCL thickness and letter read (total GCL: $r=0.35, p=0.001$, and temporal GCL: $r=0.39, p<0.001)$. Results are shown in Table 3 .

No significant correlations were observed between ERG and structural parameters.

The amplitude of mfVEPs in the central retina was inversely correlated with the EDSS score $(r=-0.443$, $p=0.002)$. No other significant associations between functional parameters and the EDSS score were observed.

\section{Discussion}

In the present study, we evaluated visual function parameters of $48 \mathrm{MS}$ patients and 46 healthy controls using the vision monitor unit Monpack One, a multiple function stimulator device which integrates different functional, electrophysiological, and phychophysical tests for a 

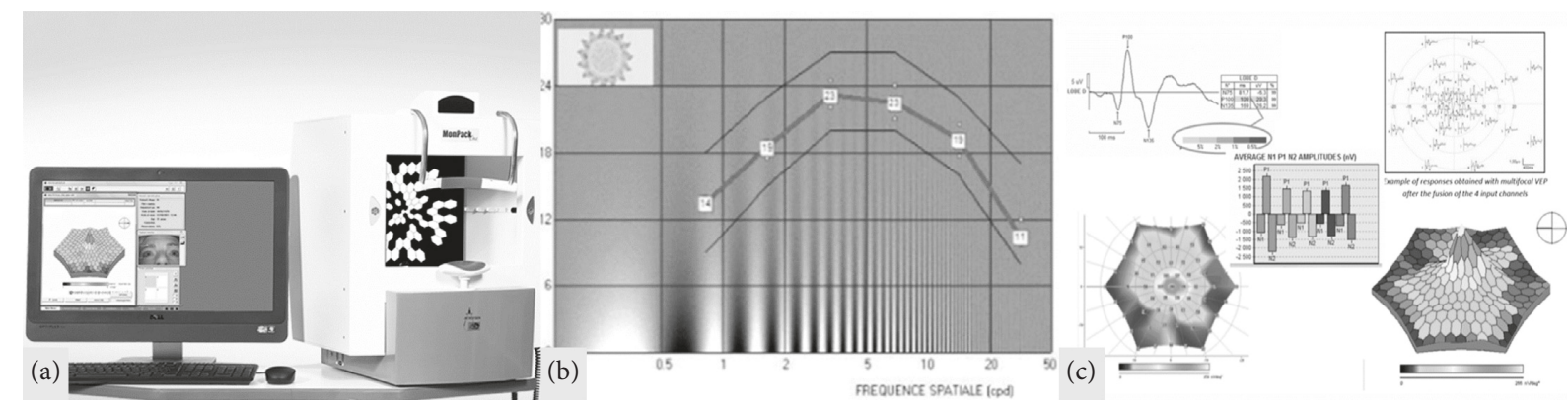

Figure 1: (a) Monpack One monitor. (b) Displayed report of the contrast sensitivity vision test in one of our controls. (c) Displayed report of multifocal visual evoked potentials in one of our controls.

TABle 3: Correlation between visual function parameters obtained with the Monpack one device and structural parameters (macular and peripapillary) obtained with Triton optical coherence tomography.

\begin{tabular}{|c|c|c|c|c|c|c|c|c|}
\hline \multirow[t]{2}{*}{ Visual function parameters } & \multicolumn{2}{|c|}{$\begin{array}{c}\text { Inner superior } \\
\text { macula }\end{array}$} & \multicolumn{2}{|c|}{$\begin{array}{c}\text { Inner inferior } \\
\text { macula }\end{array}$} & \multicolumn{2}{|c|}{$\begin{array}{l}\text { Total GCL } \\
\text { thickness }\end{array}$} & \multicolumn{2}{|c|}{$\begin{array}{l}\text { Temporal GCL } \\
\text { thickness }\end{array}$} \\
\hline & $r$ & $p$ & $r$ & $p$ & $r$ & $p$ & $r$ & $p$ \\
\hline VA $10 \%$ & 0.17 & 0.96 & 0.78 & 0.455 & 0.18 & 0.079 & 0.26 & 0.010 \\
\hline Letter read & 0.37 & $<0.001$ & 0.27 & 0.009 & 0.35 & 0.001 & 0.39 & $<0.001$ \\
\hline CSV 0.5 & 0.18 & 0.083 & 0.09 & 0.370 & 0.15 & 0.131 & 0.20 & 0.053 \\
\hline CSV 1 & 0.27 & 0.007 & 0.18 & 0.069 & 0.23 & 0.023 & 0.20 & 0.048 \\
\hline CSV 2 & 0.34 & 0.001 & 0.25 & 0.013 & 0.23 & 0.024 & 0.25 & 0.016 \\
\hline CSV 5 & 0.36 & $<0.001$ & 0.25 & 0.016 & 0.27 & 0.008 & 0.33 & 0.001 \\
\hline CSV 10 & 0.33 & 0.001 & 0.30 & 0.003 & 0.28 & 0.007 & 0.39 & $<0.001$ \\
\hline CSV 20 & 0.25 & 0.014 & 0.26 & 0.011 & 0.13 & 0.135 & 0.25 & 0.013 \\
\hline Avg. deficit & -0.27 & 0.008 & -0.22 & 0.034 & -0.27 & 0.008 & -0.34 & 0.001 \\
\hline Corrected avg. deficit & -0.30 & 0.003 & -0.26 & 0.011 & -0.32 & 0.002 & -0.36 & $<0.001$ \\
\hline Variance of deficits & -0.35 & 0.001 & -0.29 & 0.004 & -0.25 & 0.017 & -0.28 & 0.005 \\
\hline Temporal fluctuation & -0.30 & 0.003 & -0.24 & 0.019 & -0.26 & 0.011 & -0.27 & 0.008 \\
\hline
\end{tabular}

Bold letters indicate a significant correlation. Abbreviations: VA, visual acuity; CSV, contrast sensitivity vision; avg., average; GCL, ganglion cell layer.

TABLE 4: Correlation between visual field parameters obtained with the Monpack One device and macular measurements obtained with Triton optical coherence tomography.

\begin{tabular}{lcccccccccc}
\hline \multirow{2}{*}{ Visual function parameters } & \multicolumn{2}{c}{ Central } & \multicolumn{2}{c}{ Inner superior } & \multicolumn{2}{c}{ Inner nasal } & \multicolumn{2}{c}{ Inner inferior } & \multicolumn{2}{c}{ Inner temporal } \\
& $r$ & $p$ & $r$ & $p$ & $r$ & $p$ & $r$ & $p$ & $r$ \\
\hline Avg. deficit & -0.28 & $\mathbf{0 . 0 0 7}$ & -0.27 & $\mathbf{0 . 0 0 8}$ & -0.23 & $\mathbf{0 . 0 2 5}$ & -0.22 & $\mathbf{0 . 0 3 4}$ & -0.32 \\
Corrected avg. deficit & -0.25 & $\mathbf{0 . 0 1 6}$ & -0.30 & $\mathbf{0 . 0 0 3}$ & -0.23 & $\mathbf{0 . 0 2 4}$ & -0.26 & $\mathbf{0 . 0 1 1}$ & -0.30 & $\mathbf{0 . 0 0 3}$ \\
Variance of deficits & -0.25 & $\mathbf{0 . 0 1 7}$ & -0.35 & $\mathbf{0 . 0 0 1}$ & -0.25 & $\mathbf{0 . 0 1 4}$ & -0.29 & $\mathbf{0 . 0 0 4}$ & -0.33 & $\mathbf{0 . 0 0 1}$ \\
Temporal fluctuation & -0.13 & 0.194 & -0.30 & $\mathbf{0 . 0 0 3}$ & -0.22 & $\mathbf{0 . 0 2 8}$ & -0.24 & $\mathbf{0 . 0 1 9}$ & -0.26 & $\mathbf{0 . 0 1 0}$ \\
\hline
\end{tabular}

Bold letters indicate a significance $<0.05$. Abbreviations: avg., average.

complete evaluation of visual function. To the best of our knowledge, this is the first study featuring visual function tests with Monpack One in patients with a neurodegenerative disease. Our patients presented lower VA, decreased CSV affecting medium and lower frequencies, increased defects in the visual field, and reduced amplitude in the P100 component of the multifocal VEP affecting the central and inferior sectors of the retina. All these altered parameters were observed using one single device, the vision monitor unit Monpack One.

Visual dysfunction was previously reported in MS patients and may occur in up to $80 \%$ of cases during the course of the disease [16]. Contrast sensitivity provides more complete information about visual function than visual acuity tests. Measures of low-contrast vision and CSV were sensitive to visual impairment, even in patients with VA of $20 / 20$ or better (measured with a Snellen chart), and have been correlated with poorer performances in everyday tasks $[8,17-20]$. Our patients presented reduced CSV in low and midspatial frequencies and low-contrast VA compared with healthy controls, which is consistent to previous research involving classic visual function tests $[11,21,22]$ and tests performed with other video processors [23, 24]. However, high spatial frequencies were not significantly affected in our patients, contrary to previous findings [11,24]. More studies including a larger sample size would be needed to establish whether the Monpack One device detects significant differences in CSV at high frequencies in MS.

Patients suffering from MS present visual field defects compared with healthy controls, according to previous 
published research $[25,26]$. However, other studies suggested different results $[27,28]$. Despite the fact that visual field defects are more frequently reported in patients with MS and previous history of ON [29], asymptomatic visual field disturbances seem to be present also in patients without a previous episode of ON [14].

Our patients presented significant alterations in all visual field parameters compared with healthy controls, which are consistent with results provided by Pueyo et al. and Castro et al. Since none of our patients had a previous history of $\mathrm{ON}$, our results also agree with results provided by Chorazy et al. and strengthen the evidence of visual field abnormalities in non-ON MS patients.

Electrophysiological responses of the visual pathway are also altered in patients with MS [11,30,31]. According to previous published studies, MS patients present increased latency and decreased amplitude in the P100 waveform of the pattern VEP [11, 27, 32, 33]. The pattern VEP provides information for a complete study of the visual pathway, from the optic nerve to the visual cortex. The pattern VEP has been shown to be more sensitive than perimetry, contrast sensitivity, and retinal structural defects at detecting hidden visual loss in patients with MS with 20/20 vision and without history of optic neuritis [30,34]. However, the pattern VEP is dominated by macular responses, thus other focal defects may not be detected. The multifocal VEP captures a significantly larger area of the visual field than the pattern VEP and can provide information of the global optical path, topographic assessment of amplitude and latency, and thus detects focal and peripheral defects [35]. Recent studies have shown the sensitivity of the technique in identifying multifocal VEP defects after recovery from an episode of ON [36] and also in patients suffering from MS (with and without history of ON) [37]. Our patients presented decreased amplitude of P100 waveform in the multifocal VEP (which represents neurodegeneration), especially those corresponding to central, inferonasal, and inferotemporal retina. Focal defects are not usually reported when performing pattern VEPs [11].

The pattern ERG is considered to be the most useful neurophysiologic technology for detecting abnormalities in the visual pathway caused by neurodegenerative diseases [38]. Although the exact origin of the pattern ERG is not clear, it seems to be generated by the inner layers of the central retina (ganglion cells and their axons) [11], so it reflects a more specific involvement of the retinal nerve fiber layer anomalies than the alterations detected by PEV. Previous research demonstrated increased latency of the N95 waveform of the pattern ERG in patients with MS and an increased P50/N95 ratio [11]. In the present study, MS patients presented increased latencies of the N95 waveform which is consistent with previous reports. However, this difference between patients and controls was not found to be significant, probably due to the small sample size of our study.

Structural changes in the retina of patients with MS have been widely studied. Optic nerve atrophy and thinning of the peripapillary retinal nerve fiber layer are two typical findings of patients with MS. Hitherto, studies using spectral-domain
OCT have revealed that the retina in non-ON eyes shows peripapillary thinning compared to healthy controls $[39,40]$. The most recent milestone in the development of retina and choroid visualization strategies is swept source OCT (SSOCT), with a scan speed of $100,000 \mathrm{~A}$-scans/sec, which provides more accurate three-dimensional images of the retina and the choroid than previous spectral-domain devices [41]. In our study, we used Triton SS-OCT to evaluate the retina of patients with MS. Furthermore, we analyzed the correlation between functional measurements obtained with the Monpack One monitor and structural data measured with Triton OCT. The correlation between functional changes in patients with MS [10] and other neurodegenerative diseases [42] and retinal structural measurements has been previously evaluated using spectral-domain OCT devices. To the best of our knowledge, this is the first study assessing the correlation between visual function parameters obtained with a multifunction simulator device and SS-OCT technology. Our analysis revealed that contrast sensitivity parameters and visual field data correlated most significantly with macular and peripapillary measurements. However, the strength of the association was limited. Correlations between functional and structural retinal parameters are seldom strong or perfect, since changes in structural parameters are not happening at the same time as functional changes. This is frequently observed in glaucoma and also in neurodegenerative diseases such as MS, and Parkinson electrophysiological tests performed with the Monpack One device demonstrated barely no association with obtained structural data, opposite to previous studies where significant association between structural changes and electroretinogram/ multifocal visual evoked potentials was observed $[43,44]$. Nonparametric tests (as the one used in our calculations) tests are more demanding to accept statistical differences; this also might explain why we did not find more association between parameters. Additionally, devices used in previous studies (to obtain both structural and functional data) differ from what was used in our present study; we believe results might not be entirely comparable. However, our results suggest that the correlation between functional data obtained with the multifunction simulator device and structural retinal measurements may only be applicable to CSV tests and perimetry data. In addition, there were no observable differences between MS patients and controls in the ERG measurements (opposite to previous reported data). Taken altogether, these results might suggest that the ability of the ERG function of the Monpack One monitor should be improved. However, more studies using the multifunction simulator device Monpack One are needed to corroborate our findings.

The main goal of this study was to analyze the capability of the vision monitor unit Monpack One of detecting visual dysfunction in MS. Low-contrast VA, CSV, visual field parameters, and multifocal VEPs were significantly altered in our patients, and these results agree with previous published research. Additionally, since none of our patients presented any previous episode of optic neuritis, which causes inflammation and retrograde retinal cells alteration, we are certain that results in our patients were associated 
with disease itself and not with previous inflammatory episodes of the optic nerve. Moreover, similar alterations in visual function tests in these patients have been associated with axonal loss secondary to MS $[10,11]$.

In conclusion, patients with MS and without ON antecedent present visual dysfunction detectable with the vision monitor unit Monpack One. Measurements obtained with this device correlate with structural retinal data obtained with SS-OCT technology. Monpack One may be a fast and useful tool to provide a complete evaluation of axonal damage in patients with MS, although some of the electrophysiological tests might require further improvement.

\section{Data Availability}

The data used to support the findings of this study are available from the corresponding author upon request.

\section{Ethical Approval}

This study was conducted in accordance with the guidelines established by the Ethics Committee of the Miguel Servet Hospital and based on the principles of the Declaration of Helsinki.

\section{Consent}

All subjects gave detailed consent to participate in this study.

\section{Conflicts of Interest}

The authors declare that they have no conflicts of interest.

\section{Acknowledgments}

MS was supported by a fellowship from Instituto de Salud Carlos III, Spain (Rio Hortega program: CM14/00026). Publishing and edition costs were given by the Miguel Servet Ophthalmology Research Group (Grupo de investigacion e innovación HUMS oftalmología-GIMSO).

\section{Supplementary Materials}

Supplementary Table 1: macular and peripapillary measurements (standard deviation between parenthesis) obtained with Triton swept source optical coherence tomography in patients with multiple sclerosis and healthy controls. Bold letters indicate statistical significance $(p<0.05)$. Abbreviations: MS, multiple sclerosis. (Supplementary Materials)

\section{References}

[1] E. Garcia-Martin, V. Pueyo, I. Pinilla, J.-R. Ara, J. Martin, and J. Fernandez, "Fourier-domain OCT in multiple sclerosis patients: reproducibility and ability to detect retinal nerve fiber layer atrophy," Investigative Opthalmology \& Visual Science, vol. 52, no. 7, pp. 4124-4131, 2011.

[2] J. Fisher, D. Jacobs, C. Markowitz et al., "Relation of visual function to retinal nerve fiber layer thickness in multiple sclerosis," Ophthalmology, vol. 113, no. 2, pp. 324-332, 2006.
[3] E. Gordon-Lipkin, B. Chodkowski, D. S. Reich et al., "Retinal nerve fiber layer is associated with brain atrophy in multiple sclerosis," Neurology, vol. 69, no. 16, pp. 1603-1609, 2007.

[4] E. Fisher, J.-C. Lee, K. Nakamura, and R. A. Rudick, "Gray matter atrophy in multiple sclerosis: a longitudinal study," Annals of Neurology, vol. 64, no. 3, pp. 255-265, 2008.

[5] L. K. Fisniku, D. T. Chard, J. S Jackson et al., "Gray matter atrophy is related to long-term disability in multiple sclerosis," Annals of Neurology, vol. 64, pp. 247-254, 2008.

[6] M. Filippi, M. A. Rocca, M. Calabrese et al., "Intracortical lesions: relevance for new MRI diagnostic criteria for multiple sclerosis," Neurology, vol. 75, no. 22, pp. 1988-1994, 2010.

[7] D. Pitt, A. Boster, W. Pei et al., "Imaging cortical lesions in multiple sclerosis with ultra-high-field magnetic resonance imaging," Archives of Neurology, vol. 67, pp. 812-818, 2010.

[8] L. J. Balcer and E. M. Frohman, "Evaluating loss of visual function in multiple sclerosis as measured by low-contrast letter acuity," Neurology, vol. 74, no. 3, pp. 16-23, 2010.

[9] P. Villoslada, A. Cuneo, J. Gelfand, S. L. Hauser, and A. Green, "Color vision is strongly associated with retinal thinning in multiple sclerosis," Multiple Sclerosis Journal, vol. 18, no. 7, pp. 991-999, 2012.

[10] M. Satue, M. J. Rodrigo, S. Otin et al., "Relationship between visual dysfunction and retinal changes in patients with multiple sclerosis," PLoS One, vol. 11, no. 6, Article ID e0157293, 2016.

[11] D. Rodriguez-Mena, C. Almarcegui, I. Dolz et al., "Electropysiologic evaluation of the visual pathway in patients with multiple sclerosis," Journal of Clinical Neurophysiology, vol. 30, no. 4, pp. 376-381, 2013.

[12] S. A. Trip, P. G. Schlottmann, S. J. Jones et al., "Retinal nerve fiber layer axonal loss and visual dysfunction in optic neuritis," Annals of Neurology, vol. 58, no. 3, pp. 383-391, 2005.

[13] G. E. Holder, "Pattern electroretinography (PERG) and an integrated approach to visual pathway diagnosis," Progress in Retinal and Eye Research, vol. 20, no. 4, pp. 531-561, 2001.

[14] M. Chorazy, W. Drozdowski, N. Sherkawey, and Z. Mariak, "Asymptomatic visual field disturbances in multiple sclerosis patients without a history of optic neuritis," Neurologia $i$ Neurochirurgia Polska, vol. 41, no. 3, pp. 223-228, 2007.

[15] C. H. Polman, S. C. Reingold, G. Edan et al., "Diagnostic criteria for multiple sclerosis: 2005 revisions to the "McDonald Criteria"," Annals of Neurology, vol. 58, no. 6, pp. 840-846, 2005.

[16] W. I. MacDonald and D. Barnes, "The ocular manifestation of multiple sclerosis. 1. Abnormalities in the afferent visual system," Journal of Neurology, Neurosurgery \& Psychiatry, vol. 55, no. 9, pp. 747-752, 1992.

[17] B. Ashworth, P. A. Aspinall, and J. D. Mitchell, "Visual function in multiple sclerosis," Documenta Ophthalmologica, vol. 73, no. 3, pp. 209-224, 1989.

[18] I. Bodis-Wollner and J. R. Brannan, "Hidden visual loss in optic neuropathy is revealed using Gabor patch contrast perimetry," Journal of Clinical Neuroscience, vol. 4, no. 5, pp. 284-291, 1997.

[19] E. M. Mowry, M. J. Loguidice, A. B. Daniels et al., "Vision related quality of life in multiple sclerosis: correlation with new measures of low and high contrast letter acuity," Journal of Neurology, Neurosurgery, and Psychiatry, vol. 80, no. 7, pp. 767-772, 2009.

[20] S. J. Leat, G. E. Legge, and M. A. Bullimore, "What is low vision? A re-evaluation of definitions," Optometry and Vision Science, vol. 76, no. 4, pp. 198-211, 1999. 
[21] J. Schinzel, H. Zimmermann, F. Paul et al., "Relations of low contrast visual acuity, quality of life and multiple sclerosis functional composite: a cross-sectional analysis," $B M C$ Neurology, vol. 14, no. 1, p. 31, 2014.

[22] A. Soler García, A. González Gómez, L. C. Figueroa-Ortiz, A. García-Ben, and J. García-Campos, "Relación entre el test de sensibilidad al contraste y el nivel de gravedad en pacientes con esclerosis múltiple," Archivos de la Sociedad Española de Oftalmología, vol. 89, no. 9, pp. 347-351, 2014.

[23] J. G. Vieira-Gutemberg, L. C. Mendes-Santos, M. K. CavalcantiGaldino, N. A. Santos, and M. L. De Bustamante Simas, "Contrast sensitivity in relapsing-remitting multiple sclerosis assessed by sine-wave gratings and angular frequency stimuli," Visual Neuroscience, vol. 31, no. 6, pp. 381-386, 2014.

[24] M. Owidzka, M. Wilczynski, and W. Omulecki, "Evaluation of contrast sensitivity measurements after retrobulbar optic neuritis in multiple sclerosis," Graefe's Archive for Clinical and Experimental Ophthalmology, vol. 252, no. 4, pp. 673-677, 2014.

[25] V. Pueyo, J. R. Ara, C. Almarcegui et al., "Sub-clinical atrophy of the retinal nerve fibre layer in multiple sclerosis," Acta Ophthalmologica, vol. 88, no. 7, pp. 748-752, 2010.

[26] S. M. C. Castro, A. Damasceno, B. P. Damasceno et al., "Visual pathway abnormalities were found in most multiple sclerosis patients despite history of previous optic neuritis," Arquivos de Neuro-Psiquiatria, vol. 71, no. 7, pp. 437-441, 2013.

[27] R. Herrero, E. Garcia-Martin, C. Almarcegui et al., "Progressive degeneration of the retinal nerve fiber layer in patients with multiple sclerosis," Investigative Opthalmology \& Visual Science, vol. 53, no. 13, pp. 8344-8349, 2012.

[28] E. García-Martín, V. Pueyo, J. Martin et al., "Progressive changes in the retinal nerve fiber layer in patients with multiple sclerosis," European Journal of Ophthalmology, vol. 20, no. 1, pp. 167-173, 2010.

[29] H. Cheng, M. Laron, J. S. Schiffman, R. A. Tang, and L. J. Frishman, "The relationship between visual field and retinal nerve fiber layer measurements in patients with multiple sclerosis," Investigative Opthalmology \& Visual Science, vol. 48, no. 12, pp. 5798-5805, 2007.

[30] A. Chilińska, M. Ejma, A. Turno-Kręcicka, K. Guranski, and M. Misiuk-Hojlo, "Analysis of retinal nerve fibre layer, visual evoked potentials and relative afferent pupillary defect in multiple sclerosis patients," Clinical Neurophysiology, vol. 127, no. 1, pp. 821-826, 2016.

[31] N. Chirapapaisan, S. Laotaweerungsawat, W. Chuenkongkaew et al., "Diagnostic value of visual evoked potentials for clinical diagnosis of multiple sclerosis," Documenta Ophthalmologica, vol. 130, no. 1, pp. 25-30, 2015.

[32] V. Pueyo, J. Martin, J. Fernandez et al., "Axonal loss in the retinal nerve fiber layer in patients with multiple sclerosis," Multiple Sclerosis Journal, vol. 14, no. 5, pp. 609-614, 2008.

[33] F. C. Gundogan, S. Demirkaya, and G. Sobaci, "Is optical coherence tomography really a new biomarker candidate in multiple sclerosis? A structural and functional evaluation," Investigative Opthalmology \& Visual Science, vol. 48, no. 12, pp. 5773-5781, 2007.

[34] H. A. M. Van Diemen, P. Lanting, J. C. Koetsier, R. L. M. Strijers, H. K. Van Walbeek, and C. H. Polman, "Evaluation of the visual system in multiple sclerosis: a comparative study of diagnostic tests," Clinical Neurology and Neurosurgery, vol. 94, no. 3, pp. 191-195, 1992.

[35] A. Klistorner, H. Arvind, T. Nguyen et al., "Multifocal VEP and OCT in optic neuritis: a topographical study of the structure- function relationship," Documenta Ophthalmologica, vol. 118, no. 2, pp. 129-137, 2009.

[36] A. Klistorner, C. Fraser, R. Garrick, S. Graham, and H. Arvind, "Correlation between full-field and multifocal VEPs in optic neuritis," Documenta Ophthalmologica, vol. 116, no. 1, pp. 19-27, 2008.

[37] M. Laron, H. Han Cheng, B. Bin Zhang, J. S. Schiffman, R. A. Tang, and L. J. Frishman, "Comparison of multifocal visual evoked potential, standard automated perimetry and optical coherence tomography in assessing visual pathway in multiple sclerosis patients," Multiple Sclerosis Journal, vol. 16, no. 4, pp. 412-426, 2010.

[38] G. Corallo, S. Cicinelli, M. Papadia, F. Bandini, A. Uccelli, and G. Calabria, "Conventional perimetry, short-wavelength automated perimetry, frequency-doubling technology, and visual evoked potentials in the assessment of patients with multiple sclerosis," European Journal of Ophthalmology, vol. 15, no. 6, pp. 730-738, 2005.

[39] J. Dorr, K. D. Wernecke, M. Bock et al., "Association of retinal and macular damage with brain atrophy in multiple sclerosis," PLoS One, vol. 6, no. 4, Article ID e18132, 2011.

[40] J. J. González-López, G. Rebolleda, M. Leal et al., "Comparative diagnostic accuracy of ganglion cell-inner plexiform and retinal nerve fiber layer thickness measures by Cirrus and Spectralis optical coherence tomography in relapsing-remitting multiple sclerosis," BioMed Research International, vol. 2014, Article ID 128517, 10 pages, 2014.

[41] S. Copete, I. Flores-Moreno, J. A. Montero, J. S. Duker, and J. M. Ruiz-Moreno, "Direct comparison of spectral-domain and swept-source OCT in the measurement of choroidal thickness in normal eyes," British Journal of Ophthalmology, vol. 98, no. 3, pp. 334-338, 2014.

[42] V. Polo, M. Satué, E. García Martín, and L. Pablo, "Visual dysfunction and its correlation with retinal changes in patients with Parkinson disease," BMJ Open, vol. 6, no. 5, Article ID e009658, 2016.

[43] C. Almarcegui, I. Dolz, V. Pueyo et al., "Correlation between functional and structural assessments of the optic nerve and retina in multiple sclerosis patients," Neurophysiologie Clinique/Clinical Neurophysiology, vol. 40, no. 3, pp. 129-135, 2010.

[44] P. Sriram, C. Wang, C. Yiannikas et al., "Relationship between optical coherence tomography and electrophysiology of the visual pathway in non-optic neuritis eyes of multiple sclerosis patients," PLoS One, vol. 9, no. 8, Article ID e102546, 2014. 


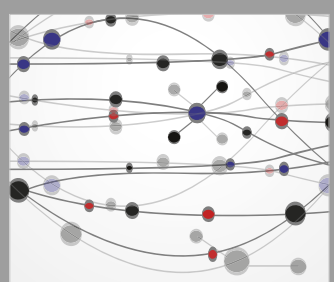

The Scientific World Journal
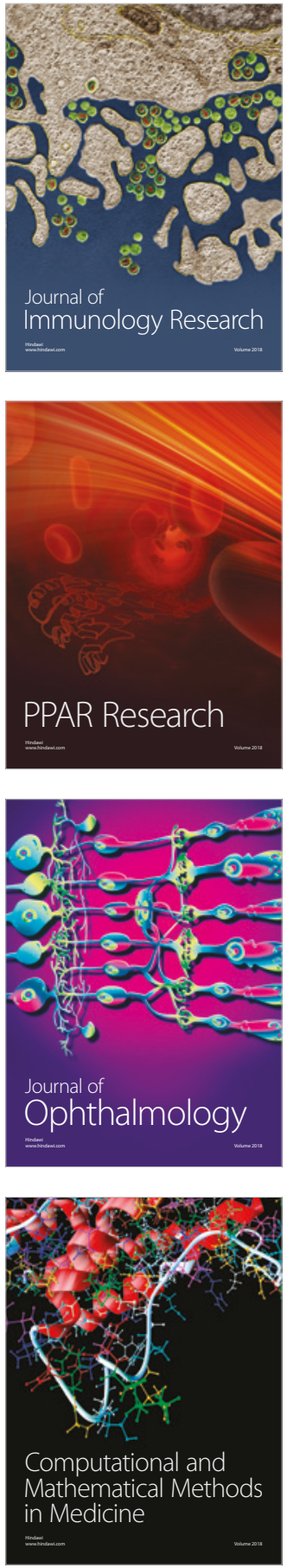

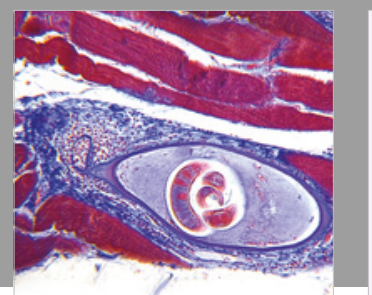

Gastroenterology Research and Practice

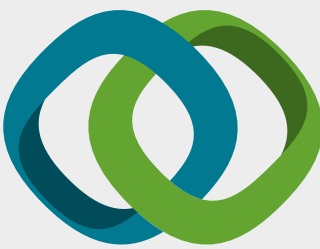

\section{Hindawi}

Submit your manuscripts at

www.hindawi.com
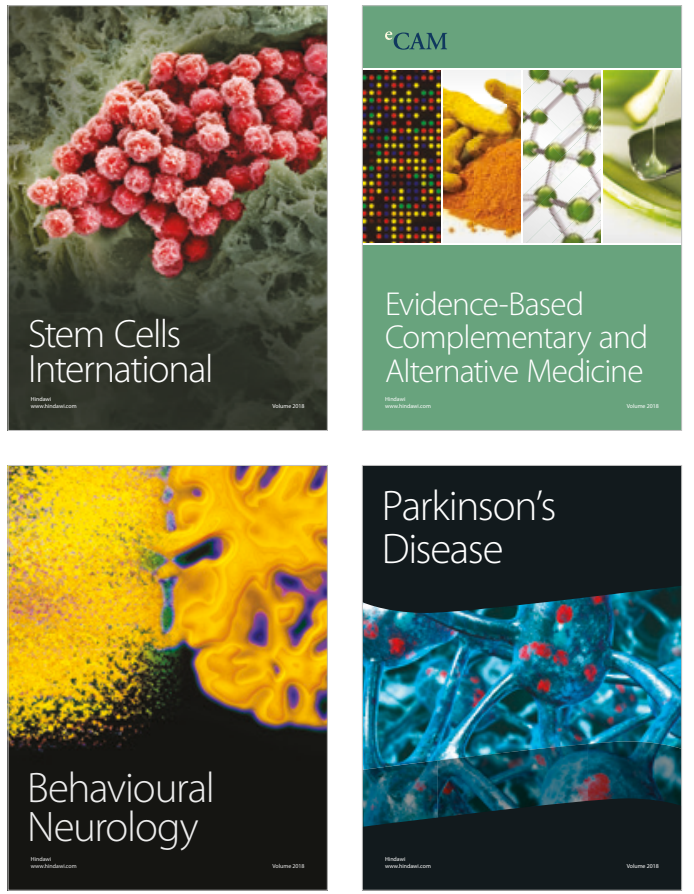

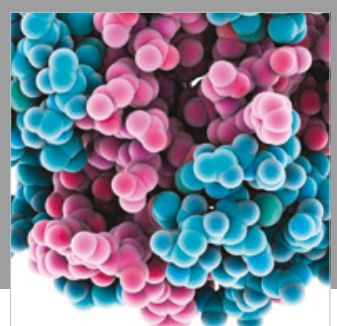

ournal of

Diabetes Research

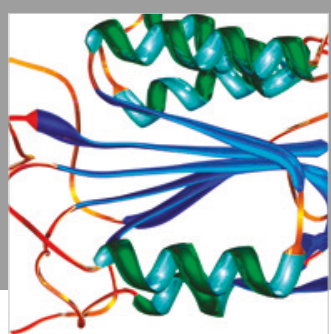

Disease Markers
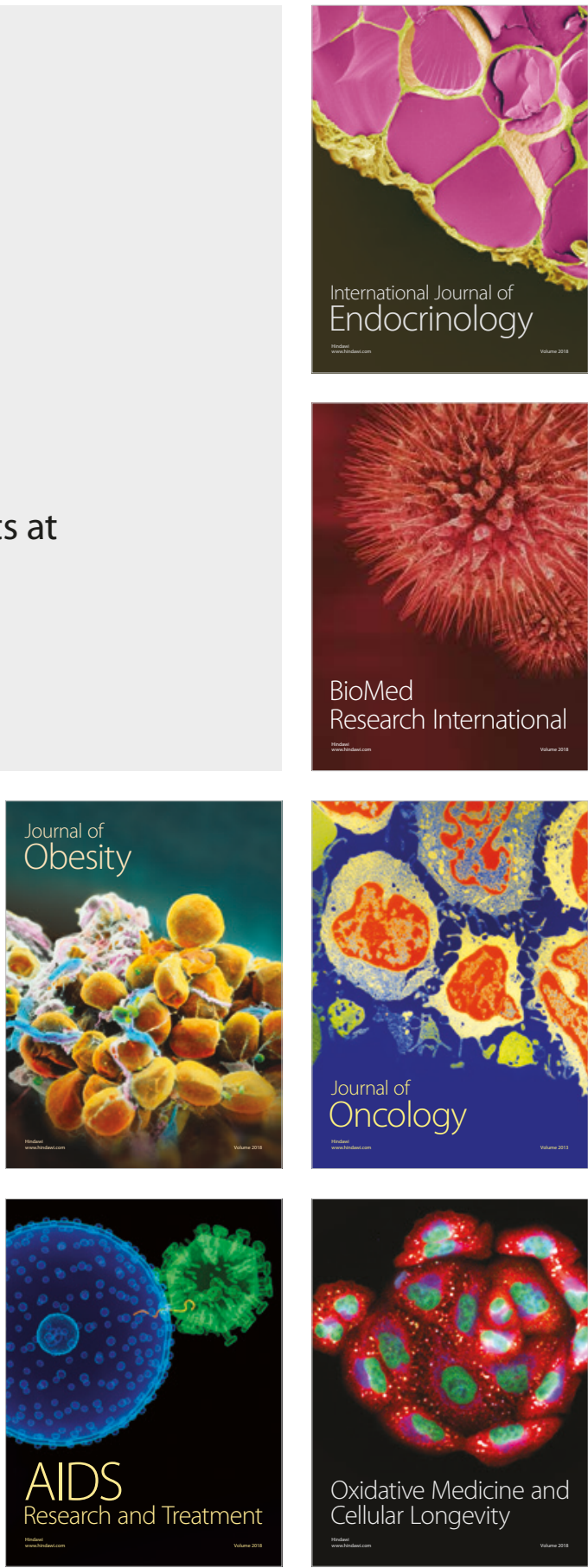